associated significance testing (Duffy et al, 2005). The evaluation was also limited by the absence of a comparison group which did not receive the e-supervision. Therefore, positive outcomes cannot be confidently attributed solely to the esupervision programme. The study design, which relied heavily on the direct report of participants, could have been strengthened by including additional objective measures of outcome, such as a grading of the change in confidence of the GPs as assessed by the supervising psychiatrist following case-based discussions. Although the evaluation attempted to assess the overall standardisation of e-supervision sessions through the semi-structured interview questions about time and use of the WHO mhGAP-IG, no evaluation data were collected about the degree of standardisation of individual e-supervision sessions of all participants. A lack of standardisation in the execution of e-supervision sessions may have affected the outcomes of the programme. A future trial of the programme should include more stringent controls of quality and standardisation.

In summary, the limited findings of this small pilot do not confirm whether the mhGAP-IG can be used effectively to supervise primary care doctors at a distance. It is recommended that the barriers to the e-supervision programme are addressed fully. It would then be appropriate to re-evaluate the mhGAP e-supervision programme with a larger number of GPs and psychiatrists. Ideally, this evaluation should follow a controlled trial design and include a detailed economic evaluation. This needs to take place before the esupervision programme can be confidently rolled out to other countries.

\section{References}

Ali, S., Saeed, K. \& Hughes, P. (2012) Evaluation of a mental health training project in the Republic of the Sudan using the Mental Health Gap Action Programme curriculum. International Psychiatry, 9, 43-45.

Bruckner, T., Scheffler, R., Shen, G., et al (2011) The mental health workforce gap in low- and middle-income countries: a needs-based approach. Bulletin of the World Health Organization [online], 89. (accessed 11 October 2014).

Duffy, M., Munro, B. \& Jacobsen, B. (2005) Key principles of statistical inference. In Statistical Methods for Health Care Research. (5th edn) (ed. B. Munro), pp. 73-105. Lippincott, Williams \& Wilkins. Heckner, C. \& Giard, A. (2005) A comparison of on-site and telepsychiatry supervision. Journal of the American Psychiatric Nurses Association, 11, 35-38.

Julyan, T. E. (2009) Educational supervision and the impact of workplace-based assessments: a survey of psychiatry trainees and their supervisors. BMC Medical Education, 9, 51.

Kakuma, R., Minas, H., van Ginneken, N., et al (2011) Human resources for mental health care: current situation and strategies for action. Lancet, 378, 1654-1663.

Kuehn, B. (2007) Global shortage of health workers, brain drain stress developing countries. JAMA, 298, 1853-1855.

Rahman, A., Nizami, A., Minhas, A., et al (2006) E-mental health in Pakistan: a pilot study of training and supervision in child psychiatry using the internet. Psychiatric Bulletin, 30, 149-152.

WHO (2005) Mental Health Atlas. Word Health Organization. WHO (2010) Mental Health Gap Action Programme (mhGAP): Scaling Up Care for Mental, Neurological and Substance Abuse Disorders. World Health Organization.

WHO (2011) Mental Health Atlas. World Health Organization.

World Bank (2014) Sudan. At http://data.worldbank.org/country/ sudan (accessed 12 July 2014).

\section{RESEARCH} PAPER

\title{
The WHO mhGAP Intervention Guide for people with intellectual disability: the Sri Lankan experience
}

\author{
Rohit Gumber, ${ }^{1}$ Shweta Gangavati, ${ }^{2}$ Sabyasachi Bhaumik, ${ }^{3}$ Sherva Cooray, ${ }^{4}$ \\ Kiran Purandare, ${ }^{5}$ Jayan Mendis ${ }^{6}$ and Chamila Abeywickrema ${ }^{7}$
}

${ }^{1}$ Consultant Psychiatrist, Leicester Frith Hospital, Leicester, UK, email gumber@doctors.org.uk

${ }^{2}$ Locum Speciality Doctor, Leicester Frith Hospital, Leicester, UK

${ }^{3}$ Consultant Psychiatrist, Leicester Frith Hospital, Leicester, UK, and Honorary Chair, University of Leicester, UK

Leicester, UK

${ }^{4}$ Consultant Psychiatrist and Chair of the ICD-11/DID Working Group, Faculty of Psychiatry of Intellectual Disability, Royal College of

Psychiatrists, London, UK

${ }^{5}$ Consultant Psychiatrist, C\&NW London NHS Foundation Trust, UK

${ }^{6}$ Director and Consultant

Psychiatrist, National Institute of Mental Health, Colombo, Sri Lanka

${ }^{7}$ Medical Officer, National Institute of Mental Health, Colombo, Sri Lanka
In recognition of the treatment gap in mental health, the World Health Organization (WHO) Mental Health Gap Action Programme (mhGAP) was launched in 2008 and has proved successful. The paper describes the launch of the first mhGAP intellectual disabilities pilot project in Sri Lanka. It reports on the development of the materials and the key lessons learnt.

Mental disorders and intellectual disability are universal, but many low-income countries are unable to provide the appropriate healthcare services. This results in a significant treatment gap. It is estimated that $76-85 \%$ of patients with mental disorders do not receive the treatment they need (Khon et al, 2004). Some cultures emphasise the role of religion in understanding intellectual disability and caring for people with such conditions. This population is then less likely to seek help from medical professionals (Royal College of Psychiatrists, 2011). According to the World Health Organization (WHO), within low- and middleincome countries (LMICs) 80\% of all people with a disability live in poverty in isolated rural areas, often stigmatised and, despite their high rates of morbidity, have poor access to healthcare (WHO, 2009). The WHO (2011) estimated a shortage of 55000 psychiatrists and over 600000 mental health nurses worldwide. 
The presence of services alone does not guarantee access to them. The WHO (2007) concluded that geographical factors and the socioeconomic status of the persons needing care affected whether services were accessed. These appear to have greater impact within LMICs. Furthermore, the use of clinical guidelines that establish minimum standards to ensure proper care and interventions for persons with intellectual disability is lower in these countries. The challenge to meet this need calls for innovative solutions.

In recognition of this treatment gap the WHO launched the Mental Health Gap Action Programme (mhGAP) in 2008. It aims to support the scaling up of services for mental, neurological and substance use disorders (WHO, 2008). The programme emphasises the delivery of pharmacological and psychosocial interventions in non-specialised healthcare settings. The importance of geographical factors in access to care for patients with intellectual disability highlights the need for a model to support non-specialist workers to deliver healthcare in rural settings. The mhGAP has been implemented in Ethiopia, Jordan, Panama and Nigeria, and more countries have expressed an interest. It is available in a number of languages, including French, Arabic, Russian and Chinese (Saxena, 2011).

There is a substantial amount of literature on the implementation of the programme for the general population but not for those with intellectual disability. Abdulmalik et al (2013) describe the implementation of mhGAP for the general population in Nigeria, using a key component of its delivery - the Intervention Guide (IG). This is a manual with guidance to facilitate the recognition and evidence-based management of common conditions. Key lessons learnt from this work include the importance of engaging local stakeholders early and acknowledging the gaps in both services and the knowledge of primary care workers. The project emphasised the importance of experiential teaching methods, such as role-play and group work. The importance of integration into primary care with good working relationships between primary health workers, general practitioners and psychiatrists was also highlighted, with the provision of ongoing supervision being an important factor to ensure sustainability of the programme.

The current mhGAP training does not address the mental health needs that are encountered among people with intellectual disability. Bearing this in mind, the International Links working group on intellectual disability of the Royal College of Psychiatrists developed modules on intellectual disability (mhGAP-ID modules) which were first piloted in Sri Lanka. Once finalised, the aim was to deliver them conjointly with mhGAP training in order to maximise resources and output.

\section{The Sri Lankan context}

Sri Lanka has a population of 19 million. None of the country's 48 consultant psychiatrists are specialists in intellectual disability. In addition, there are a number of psychiatrists in training and medical officers for mental health (MOMH), who have received around 6-12 months of training prior to deployment (Jenkins et al, 2012). The WHO (2011) estimated the requirement for psychiatrists to be $1.62 / 100000$ population by 2015 , well above the current level of provision. Additionally, there is a shortage of over 3000 mental health nurses and over 1500 psychosocial care providers. Psychiatric care is mostly provided from the capital, Colombo. Community services are limited to governmentrun primary care dispensaries, supplemented by some private providers of primary care (Jenkins et al, 2012).

Sri Lanka benefits from diverse forms of traditional medical treatment for physical and mental illnesses, from ancient times (Beiser et al, 2003; Fernando \& Weerackody, 2009). The Mahavamsa (originally written in Pali between the 6th century BC and the 4th century AD) suggests Sri Lanka was the first country in the world to have established dedicated hospitals (see http://mahavamsa.org).

The two principal ethnic groups in the country are the Sinhalese, who tend to be Buddhist and constitute approximately $74 \%$ of the population, and the Tamil minority, who are mainly Hindu and mostly reside in the north and east. Psychological interventions now popular in the West such as meditation, mindfulness and elements of cognitive-behavioural therapy have been used in Buddhist and Hindu traditions for centuries. Sri Lanka has a complex social, religious and political history that has resulted in its population having a rich mix of Sinhalese, Tamil, Muslim, Buddhist, Islam, Hindu and Christian beliefs, as well as the influence of colonisation. Sri Lankan beliefs, knowledge and practices in relation to mental health include rituals and performances, meditation, bodhi pooja (veneration of the peepul tree), yoga, astrology, palmistry, healing through spirits, exorcism, thovil (ritual dancing and exorcism), charms and amulets (Fernando \& Weerackody, 2009). Community members often seek traditional medicine first and attend community-based mental health services only when the traditional methods fail, thereby delaying diagnosis and treatment. Many Sri Lankan families hide mental illness from society to avoid stigma and discrimination. In addition, the experience of mental illness is widely considered to be the result of either fate or blame. A general lack of mental health literacy among patients, friends and families is also reported. These factors lead to a lack of appropriate healthseeking behaviours, which is a major barrier to the provision of mental health services within Sri Lanka, as in other countries. Even when help has been sought, evidence shows that there is poor reintegration into families following treatment at a psychiatric institution in Sri Lanka (Samarasekara et al, 2012).

The National Institute of Mental Health (NIMH) in Angoda, Colombo, is a state-run hospital functioning under the Ministry of Health. This highly acclaimed and award-winning 
hospital is now Sri Lanka's largest tertiary care institution caring for patients with mental illness. The NIMH has a total of around 1500 beds and provides care for a range of mental illnesses and intellectual disability. Annually over 8000 patients are admitted to the NIMH, which provides acute and intermediate care, as well as specialised services. The main psychiatric hospital comprises 16 wards, including perinatal, forensic and intellectual disability wards. There is a separate unit for children with intellectual disability. That unit is well resourced, with space for family members and a good range of daytime activities. Adults with intellectual disability are normally admitted to general adult wards for treatment. The Health Education Unit of the hospital was relaunched as the Mental Health Training Unit in 2006 with full multimedia facilities.

\section{Project aims and objectives}

Following guidance from the WHO regarding implementation of mhGAP, local health training needs, including those in intellectual disability, were identified by the stakeholders. Training in this area is very limited for medical and nursing staff within the hospital and this limited understanding of intellectual disability, and the lack of specialist psychiatrists to supervise and support training, leads to a lack of confidence when managing psychiatric disorders in people with intellectual disability.

Over the past decade, annual training programmes were conducted by the Volunteers from Sri Lanka Psychiatric Association (SLPA) UK, coordinated by the Learning Disability Fund, in partnership with the NIMH and Sri Lanka College of Psychiatrists. At the request of the Director of the NIMH, Dr Jayan Mendis, we adapted the mhGAP for use with people with intellectual and developmental disorders. This followed the successful completion of a 2-year training programme on integrating mental health into primary care in collaboration with key stakeholders in Sri Lanka (Jenkins et al, 2012). This paper describes the launch of the 'training the trainers' pilot project, adapted from the mhGAP programme with permission from the $\mathrm{WHO}$, by the International Links working group on intellectual disability. The project was supported by the World Psychiatric Association and the Royal College of Psychiatrists, UK.

Sri Lanka has universal free provision of health services and this provided an excellent

\section{Box 1. Objectives of the mhGAP-ID pilot}

- Model teaching skills required to deliver and cascade mhGAP-ID training

- Introduce and develop the key concepts of mhGAP to an audience of mental health doctors

- Teaching/training in skills, including communication

- Introduce and develop role-play as a core teaching methodology

- Generate interest for research projects within the country

- Generate enthusiasm for further training and teaching opportunity to pilot the mhGAP model with a medical workforce, as it allowed informed refinement and development before delivery to other healthcare professionals and non-specialists. The 5 -day training programme was carried out in September 2013 at the NIMH, Colombo.

The key objectives of the programme are highlighted in Box 1.

\section{Method}

The training programme was delivered to approximately 40 doctors working and training at NIMH. The audience consisted of MOMH and psychiatry registrars (doctors in higher training). The programme focused on key areas of intellectual disability psychiatry and was split into modules delivered over 5 days. Teaching modules incorporated and encouraged didactic teaching, use of role-play and audio-visual aids, and encouraged reflection on clinical practice. Culturally appropriate case scenarios and role-play were a key part of the material, which allowed trainees to put learning into practice. The modified presentation in this cultural context has to be appreciated since religious beliefs and alternative medicines play a key role in patients' and carers' treatment-seeking behaviour. All participants were presented with comprehensive teaching materials prior to the programme, which included lecture slides, role-plays and other relevant material. The programme is outlined in Table 1.

The progress of trainees was evaluated by pre- and post-course multiple-choice questions (MCQs). We assessed the strengths and weaknesses of the course using an evaluation form covering the domains of: relevance to clinical practice, educational needs, quality of teaching and group work. It also included space for other comments and recommendations.

\section{Results}

Pre- and post-course MCQ results indicated significant improvement in knowledge (average number of pre-test questions correct was $77 \%$ compared with $84 \%$ of post-test questions). The most marked improvements were for questions on psychopathology in intellectual disability (23\% improvement), communication aids for people with intellectual disability (38\%), Down syndrome (20\%) and sudden unexplained death in epilepsy $(41 \%)$.

The course was highly appreciated, averaging satisfaction ratings of $85 \%$, although there were also pragmatic suggestions for improvement. It was considered to be highly relevant to both clinical practice and educational needs (Table 2). The pre-course reading material was highly regarded. Discussions or real cases supported the knowledgebased lectures and reflection on learning was used effectively. Additional topics suggested included counselling parents, sexual relationships and marriage, and childbearing for people with intellectual disability. Social topics such as these could be delivered but would require extensive local input, as the cultural and legal frameworks should 
Table 1

The programme

\begin{tabular}{|c|c|c|c|c|c|}
\hline & Day 1 & Day 2 & Day 3 & Day 4 & Day 5 \\
\hline 09.00 & Welcome & Recap & Recap & Recap & Recap \\
\hline $\begin{array}{l}09.15 \\
(45 \mathrm{~min})\end{array}$ & Pre-test & $\begin{array}{l}\text { Mental disorders (MD)/ } \\
\text { intellectual disability (ID) } \\
\text { overview }\end{array}$ & $\begin{array}{l}\text { Autism spectrum disorders } \\
\text { and ID }\end{array}$ & Epilepsy and ID & $\begin{array}{l}\text { A toolkit for health workers } \\
\text { in the community }\end{array}$ \\
\hline $\begin{array}{l}10.15 \\
(35 \mathrm{~min})\end{array}$ & Introduction to ID & MD/ID continued & Role-play - video & Role-play & Role-play \\
\hline 10.50 & Tea break & Tea break & Tea break & Tea break & Tea break \\
\hline $\begin{array}{l}11.10 \\
(80 \mathrm{~min})\end{array}$ & Overview of ID & Role-play & $\begin{array}{l}\text { ID and problem behaviour } \\
\text { (PB) }\end{array}$ & $\begin{array}{l}\text { Sudden unexpected death } \\
\text { in epilepsy }\end{array}$ & $\begin{array}{l}\text { Workshop: Services for } \\
\text { people with ID - the art of } \\
\text { the possible }\end{array}$ \\
\hline 12.30 & Lunch & Lunch & Lunch & Lunch & Lunch \\
\hline $\begin{array}{l}13.15 \\
(150 \mathrm{~min})\end{array}$ & Communication & ID and dementia & Management of PB & Medication use in ID & Post-test \\
\hline 15.45 & Evaluation & Evaluation & Evaluation & Evaluation & Certificates \\
\hline
\end{tabular}

Table 2

Trainee feedback

\begin{tabular}{|l|l|}
\hline & $\begin{array}{l}\text { Average score } \\
\text { (1 = poor, } \\
\text { 5 = excellent) }\end{array}$ \\
\hline Relevance to clinical practice & 4.68 \\
\hline Relevance to educational needs & 4.43 \\
\hline Quality of teaching & 4.46 \\
\hline Group work and participation & 4.25 \\
\hline
\end{tabular}

be clearly understood. The final day involved work to adapt a local toolkit for the assessment of intellectual disability, and resulted in the development of a forum to move this research forward and to maintain avenues of communication between the trainers and learners.

From a trainer's perspective, the MOMH were dedicated and keen to learn, and consequently participation in role-play and group discussion was good. The language barrier posed a few difficulties, but attention to slow and clear delivery overcame this quickly. Teaching material and delivery of teaching were adapted with the support of psychiatrists with local knowledge of Sri Lanka. A good understanding of cultural aspects and local health systems is essential before embarking on teaching. In particular, role-plays must be pertinent to the local culture and should be developed with a good understanding of available local resources and workforce. The evaluation of trainees was limited to the MCQs, which did not adequately evaluate skills related to clinical practice.

\section{Discussion}

Evaluation of the mhGAP-ID course has demonstrated that it is an effective training tool. The course is iterative, with each successive programme improving from lessons learnt from the previous sessions. We are confident about the quality and relevance of training but the programme should now focus on developing a modified mhGAP-ID intervention guide to be used to train the trainers before a full roll-out within Sri Lanka and further. Future training should not only target hospitals and tertiary care centres, but involve cascading to primary care physicians, who manage intellectual disability in the community. This will facilitate early recognition and intervention of health needs within this population. In order to ensure effective delivery of teaching to primary care physicians, the mhGAP-ID programme will need to be adapted and piloted for this group. Programmes should be flexible and adaptable to local circumstances and encourage increased training in rural areas, with an emphasis on community-based treatments, where resources are scarce. Furthermore, the capacity of non-specialists and primary care should be built within these areas through the effective use of mhGAP-ID training resources.

The cultural and legal frameworks of countries should be well understood when adapting modules for other countries, as the management of health problems in intellectual disability raises social and legal issues. Fernando \& Weerackody (2009) have posited that imposing models of services based on Western notions inherent in biomedical psychiatry is not the best way forward in Sri Lanka and they underscored the relevance of the cultural and religious perspective as a key to a successful outcome. The mhGAP curriculum and teaching materials originally developed by the WHO and modified for use with people with intellectual disability were consequently further adapted in close collaboration with Sri Lankan colleagues within this context. Similarly, other mhGAP-ID trainers should have a good understanding of the local culture, resources and workforce. This could be ensured through the use of an induction programme, where trainers work with local psychiatrists to adapt materials. Furthermore, delivery methods and the approach to testing the progress of trainees should be informed by methods familiar to the local trainees. It is advisable that role-play exercises be adapted according to the understanding of the local workforce. Although knowledge can be adequately tested through MCQs, practical skills and problem-based approaches require other methods of evaluation (e.g. structured clinical examination). 
We hope to follow the success of the mhGAP programme and implement mhGAP-ID in other countries through forums such as the Royal College of Psychiatrists International Links working group on intellectual disability. Combined mhGAP and mhGAP-ID training would allow efficient use of sometimes scarce resources and opportunities.

\section{References}

Abdulmalik, J., Kola, L., Fadahunsi, W., et al (2013) Country contextualization of the Mental Health Gap Action Programme Intervention Guide: a case study from Nigeria. PLoS Med, 10, e1001501. doi:10.1371/journal.pmed.1001501.

Beiser, M., Simich, L. \& Pandalangat, N. (2003) Community in distress: mental health needs and help seeking in the Tamil community in Toronto. International Migration, 41, 233-245.

Fernando, S. \& Weerackody, C. (2009) Challenges in developing community mental health services in Sri Lanka. Journal of Health Management, 11, 195-208.

Jenkins, R., Mendis, J., Cooray, S., et al (2012) Integration of mental health into primary care in Sri Lanka. Mental Health in Family Medicine, 9, 15-24.
Khon, R., Saxena, D., Levav, I., et al (2004) The treatment gap in mental health care. Bulletin of the World Health Organization, 82, 858-864.

Royal College of Psychiatrists (2011) Minority Ethnic Communities and Specialist Learning Disability Services. RCPsych.

Samarasekara, N., Davies, M. \& Siribaddana, S. (2012) The stigma of mental illness in Sri Lanka: the perspectives of community mental health workers. Stigma Research and Action, 2(2). doi:10.5463/sra. v1i3.48

Saxena, S. (2011) News from the WHO Mental Health Gap Programme (mhGAP). World Health Organization mhGAP Newsletter, 11 June.

WHO (2007) Atlas: Global Resources for Persons with Intellectual Disabilities. World Health Organization.

WHO (2008) mhGAP Mental Health Gap Action Programme: Scaling Up Care for Mental, Neurological, and Substance Use Disorder. World Health Organization.

WHO (2009) ECOSOC Meeting: Mental Health, Poverty and Development (Discussion Paper). World Health Organization.

WHO (2011) Human Resources for Mental Health: Workforce Shortages in Low- and Middle-Income Countries (Human Resources for Health Observer No. 8). World Health Organization.
RESEARCH PAPER

\section{Acceptability and challenges of} implementing the NICE guidelines for schizophrenia in Lagos, Nigeria

\author{
Adeniran Okewole, ${ }^{1}$ Mobolaji Dada, ${ }^{2}$ Adefemi Adeoye, ${ }^{3}$ Kehinde Lawal ${ }^{4}$ and \\ Taiwo Oduguwa ${ }^{5}$
}

${ }^{1}$ Consultant Psychiatrist Neuropsychiatric Hospital, Aro, Abeokuta, Nigeria, emai niranokewole@gmail.com

${ }^{2}$ Consultant Psychiatrist, Ekiti State University Teaching Hospital, Ekiti, Nigeria

${ }^{3}$ Consultant Psychiatrist, Federal Neuropsychiatric Hospital, Yaba Lagos, Nigeria

${ }^{4}$ Senior Registrar, Centre for Child and Adolescent Mental Health,

University College Hospital,

Ibadan, Nigeria

${ }^{5}$ Consultant Psychiatrist, Federal Neuropsychiatric Hospital, Yaba, Lagos, Nigeria
This study aimed to examine the suitability and challenges of implementing in a Nigerian tertiary mental health facility the guidelines for the management of schizophrenia produced by the UK National Institute for Health and Care Excellence (NICE). The study was based on a group discussion at the Psychiatric Hospital, Yaba, Lagos. A panel of ten individuals (consultants, senior registrars, senior psychologists and senior social workers and nurses) discussed the guidelines, having been given ample time to study the document. Five patients were also interviewed. Some of the recommendations of the NICE guidelines are already being practised in the hospital to various extents. Full implementation would be hampered by a shortage of human resources and financial constraints. The guidelines need to factor in sociocultural differences. The NICE guidelines with modifications are suitable for use in a Nigerian setting.

Clinical guidelines aim to assist health workers and service users in making effective decisions about the management of specific clinical conditions. They strive to implement available evidence and bridge the gap between research and practice (Gray, 2005). Ample evidence exists about the usefulness of guidelines. Grimshaw \& Russell (1993) did a systematic review of 59 published evaluations of clinical guidelines, of which 55 detected significant improvement in the process of care after the guidelines were introduced.

The guidelines for schizophrenia produced by the National Institute for Health and Care Excellence (NICE; formerly the National Institute for Clinical Excellence) were developed through collaboration between professionals, service users and carers (NICE, 2002, 2009). They address major treatments and services for people with schizophrenia, are evidence-based and each recommendation is graded according to level of evidence (Gray, 2005). The guidelines divide the treatment and management of schizophrenia into three phases: initiation of treatment of the first episode; acute phase; and promotion of recovery. They recommend collaboration with service users and carers in each phase. The use of care notes and care plans is also encouraged. Crisis resolution, home-treatment teams, early-intervention teams, community mental health teams and acute day hospitals are recommended, with in-patient treatment if necessary. The treatment package 